\title{
EXTENDING CERTAIN TRANSFORMATION GROUP ACTIONS IN SEPARABLE, INFINITE-DIMENSIONAL FRECHET SPACES AND THE HILBERT CUBE
}

\author{
BY JAMES E. WEST ${ }^{1}$
}

Communicated by R. D. Anderson, May 2, 1968

A theorem of V. L. Klee, Jr. [9] asserts that any homeomorphism between two compact sets in a separable, infinite-dimensional Hilbert space can be extended to a homeomorphism of the entire space onto itself. Since it has recently been shown ([1], [5] and [6]) that all separable, infinite-dimensional Frechet spaces are homeomorphic (a Frechet space being a metrizable, complete, locally convex linear topological space), this result holds in all such spaces. A salient property of compact sets in such spaces is that for each nonnull, homotopically trivial open set $U$, the relative complement in $U$ of a compact set is also nonnull and homotopically trivial (see [3, §\$3-5]). This property has been called "property $Z$ " by R. D. Anderson, who has extended Klee's theorem above to the following in [3].

Theorem A (ANDERson). If $X$ is a separable, infinite-dimensional Frechet space or the Hilbert cube, then any homeomorphism between two closed subsets of $X$ which have property $Z$ can be extended to a homeomorphism of $X$ onto itself.

It might be noted here that any compact metric space can be imbedded in the Hilbert cube as a set with property $Z$ and that any separable, complete, metric space can be imbedded in any separable, infinite-dimensional Frechet space as a closed subset with property $Z$ (see [3]).

If $X$ is a separable, infinite-dimensional Frechet space or the Hilbert cube, and if $Y$ is a closed subset of $X$ with property $Z$, then Theorem A provides a function $F$ from $H(Y)$, the group of homeomorphisms of $Y$ onto itself, into $H(X)$ such that for $h$ in $H(Y)$, $F(h) \mid Y=h$. This function is not, in general, a homomorphism, for the extension procedure does not commute with composition. It is, however, in many cases possible to construct such a homomorphism and to make it continuous in the sense of transformation groups through a linearization procedure suggested by work of P. C. Baayen and J. de Groot [4]. That is the purpose of this paper. (A transformation group is a triple $(G, X, v)$, where $G$ is a topological group, $X$ is a topological space, and $v$ is a continuous function from $G \times X$ into $X$ such that

\footnotetext{
${ }^{1}$ Supported by National Science Foundation Grant GP 7952 X.
} 
(1) for $g_{1}$ and $g_{2}$ in $G, v\left(g_{1} g_{2}, x\right)=v\left(g_{1}, v\left(g_{2}, x\right)\right)$ for each $x$ in $X$, and

(2) if $e$ is the identity of $G$, then for each $x$ in $X, v(e, x)=x$. The function $v$ is called the action of $G$ on $X$ as a transformation group.) The first theorem concerns the Hilbert cube.

THEOREM 1. Any transformation group action of a topological group $G$ on a closed subset of the Hilbert cube with property $Z$ can be extended to an action of $G$ on the Hilbert cube as a transformation group.

Proof. Let $(G, X, v)$ be the given transformation group, and let $H(X)$ be the group of all homeomorphisms of $X$ onto itself. Under the topology of uniform convergence, $H(X)$ is a separable, metrizable, topological group, and the evaluation function is an action of $H(X)$ on $X$ as a transformation group.

Let $l_{2}$ denote the real Hilbert space of all square-summable sequences of real numbers with the norm $\left\|\left(x_{i}\right)\right\|=\left(\sum_{i=1}^{\infty} x_{i}^{2}\right)^{1 / 2}$, and let $E$ be the space of all continuous functions from $H(X)$ into $l_{2}$ with the topology of uniform convergence on compacta. Now $E$ is a Hausdorff, locally convex, linear topological space, and because $H(X)$ is metrizable, $E$ is complete [8, p. 231]. Let $f_{1}$ be an imbedding of $X$ in $l_{2}$, and let $X^{\prime}$ be the union of $f_{1}(X)$ with an infinite-dimensional compact set in $l_{2}$ disjoint from it. Let $f_{2}: X^{\prime} \rightarrow E$ be the function defined by

$$
\left(f_{2}\left(x^{\prime}\right)\right)(h)=\left\{\begin{array}{ll}
f_{1} h f_{1}^{-1}\left(x^{\prime}\right), & \text { if } x^{\prime} \in f_{1}(X) \\
x^{\prime}, & \text { if } x^{\prime} \notin f_{1}(X)
\end{array}\right\}
$$

for each $h$ in $H(X)$. It is not difficult to see that $f_{2}$ is an imbedding of $X^{\prime}$ in $E$.

Let $K$ be the closed, convex hull of $f_{2}\left(X^{\prime}\right)$ in $E$, and let $L$ be the homomorphism of $H(X)$ into the group of linear homeomorphisms of $E$ onto itself defined by $(L(g)(z))(h)=z(h g)$, for all $z$ in $E$ and all $g$ and $h$ in $H(X)$. For each $g$ in $H(X), L(g)$ carries $K$ into itself.

Let $w: H(X) \times K \rightarrow K$ be the function defined by $L$, that is, $w(g, z)$ $=(L(g))(z)$ for all $z$ in $K$ and $g$ in $H(X)$. By the construction, $w\left(g, f_{2}, f_{1}(x)\right)=f_{2} f_{1} g(x)$ for each $g$ in $H(X)$ and $x$ in $X$. Further, $w$ is an action of $H(X)$ on $K$ as a transformation group. Since the closed, convex hull of a compact set in a Hausdorff, complete, locally convex, linear, topological space is compact $[10$, p. 60], $K$ is an infinitedimensional, compact, convex set in such a space.

By a theorem of Klee [9], in order to show that $K$ is homeomorphic to the Hilbert cube, it suffices to find a countable family of continuous linear functionals on $E$ which separates points of $K$ (for then using the functionals, one may imbed $K$ linearly in $l_{2}$ and use a theorem of O.-H. Keller [7]). Such a family $P$ may be constructed on $E$ as follows: Let $\left\{h_{m}\right\}_{m>0}$ be a countable, dense set in $H(X)$, and let 
$\left\{k_{n}\right\}_{n>0}$ be the family of projection functionals of $l_{2}$ onto its coordinate axes. Let $P=\left\{p_{m n}\right\}_{m, n>0}$, where for each $z$ in $E, p_{m n}(z)=k_{n}\left(z\left(h_{m}\right)\right)$. Let the Hilbert cube $Y$ be represented as the countably infinite Cartesian product of the closed interval $[-1,1]$ with itself, and let $q_{n}$ denote the projection of $Y$ onto its $n$th coordinate space. Let $K^{\prime}=K \times[-1,1]$, and let $w^{\prime}$ be the action of $H(X)$ on $K^{\prime}$ as a transformation group defined by $w^{\prime}(g,(z, t))=(w(g, z), t)$, for $g$ in $H(X)$ and $(z, t)$ in $K^{\prime}$. Let $f_{3}$ be a homeomorphism of $K$ onto $Y$, and let $f_{4}$ be the homeomorphism of $K^{\prime}$ onto $Y$ defined by $f_{4}(z, t)=\left(t, q_{1} f_{3}(z)\right.$, $\left.q_{2} f_{3}(z), \cdots\right)$, for $(z, t)$ in $K^{\prime}$.

The function $f^{\prime}: X \rightarrow Y$ defined by $f^{\prime}(x)=f_{4}\left(f_{2} f_{1}(x), 1\right)$ is an imbedding of $X$ in $Y$ as a set with property $Z$, so by Theorem A there exists a homeomorphism $f^{\prime \prime}$ of $Y$ onto itself agreeing with $f^{\prime}$ on $X$.

If $p: G \rightarrow H(X)$ is the homomorphism induced by $v$, that is, $p(g)(x)$ $=v(g, x)$ for each $g$ in $G$ and $x$ in $X$, then it is easy to see that $p$ is continuous. Define $v^{\prime}: G \times Y \rightarrow Y$ by $v^{\prime}(g, y)=f^{\prime \prime-1} f_{4} w^{\prime}\left(p(g), f_{4}^{-1} f^{\prime \prime}(y)\right)$. Now $v^{\prime}$ is the desired action of $G$ on $Y$ extending $v$.

THEOREM 2. Any transformation group action of a topological group $G$ on a closed, locally compact subset of a separable, infinite-dimensional Frechet space $Y$ can be extended to an action of $G$ on $Y$ as a transformation group.

Proof. Let $(G, X, v)$ be the given transformation group; let $X^{\prime}$ be the one-point compactification of $X$, and let $f_{1}$ be the natural imbedding of $X$ in $X^{\prime}$. Let $v^{\prime}: G \times X^{\prime} \rightarrow X^{\prime}$ be the transformation group action of $G$ on $X^{\prime}$ defined by

$$
v^{\prime}\left(g, x^{\prime}\right)=\left\{\begin{array}{ll}
f_{1} v\left(g, f_{1}^{-1}\left(x^{\prime}\right)\right), & \text { if } x^{\prime} \in f_{1}\left(X^{\prime}\right) \\
x^{\prime}, & \text { if } x^{\prime} \in f_{1}\left(X^{\prime}\right)
\end{array}\right\}
$$

for $g$ in $G$ and $x^{\prime}$ in $X^{\prime}$. Because $X^{\prime}$ is compact and metrizable, there is an imbedding $f_{2}$ of $X^{\prime}$ in the Hilbert cube $Q$ as a set with property $Z$.

Let $v^{\prime \prime}$ be the action of $G$ on $f_{2}\left(X^{\prime}\right)$ induced from $v^{\prime}$ by $f_{2}$. By Theorem 1 , there is an extension $w$ of $v^{\prime \prime}$ to an action of $G$ on $Q$. Note that for each $g$ in $G$, $w\left(g, f_{2}\left(X^{\prime}\right)-f_{2} f_{1}(X)\right)-f_{2}\left(X^{\prime}\right)=f_{2} f_{1}(X)$. Let $Y^{\prime}=Q \times s$, where $s$ is the countably infinite Cartesian product of the real line with itself. Now $Y^{\prime}$ is homeomorphic to $s$ [2, Corollary 9.4], which is a separable, infinite-dimensional Frechet space and is hence homeomorphic to $Y$. Let $w$ be the action of $G$ on $Y^{\prime}$ as a transformation group defined by $w^{\prime}(g,(y, z))=(w(g, y), z)$, for $g$ in $G$ and $(y, z)$ in $Y^{\prime}$. Let $f_{3}$ be the imbedding of $X^{\prime}$ in $Y^{\prime}$ carrying $x^{\prime}$ to $\left(f_{2}\left(x^{\prime}\right), 0\right)$, where 0 is the origin of $s$, and denote by $x_{0}$ the point $f_{3}\left(X^{\prime}-f_{1}(X)\right)$.

By a theorem of Anderson [2, Corollary 5.5], $s$ is homeomorphic to the complement of any of its compacta, so there is a homeomorphism $f_{4}$ of $Y^{\prime}-x_{0}$ onto $Y$. Let $w^{\prime \prime}$ be the action of $G$ on $Y$ as a trans- 
formation group induced from $w^{\prime}$ by $f_{4}$. Now $f_{4} f_{3} f_{1}$ is an imbedding of $X$ in $Y$ as a closed set and $w^{\prime \prime}$ extends the action of $G$ on $f_{4} f_{3} f_{1}(X)$ induced from $v$, so in order to complete the proof it suffices to show that $X$ and $f_{4} f_{3} f_{1}(X)$ have property $Z$ in $Y$ and to apply Theorem A. However, any closed, locally compact subset of a separable, infinitedimensional Frechet space has property $Z$, and one way to see this is as follows: As all separable, infinite-dimensional Frechet spaces are homeomorphic, it suffices to consider $s$, which is homeomorphic to $Q^{0}$, the countably infinite Cartesian product of the open interval $(-1,1)$ with itself and a naturally imbedded subset of the Hilbert cube. Let $T$ be any closed, locally compact subset of $Q^{0}$. There exists a homeomorphism $h$ of $Q$ on to itself carrying $Q^{0}-T$ on to $Q^{0}$ [2, Corollary 5.4].

For any (relatively) open, nonnull, homotopically trivial set $U$ in $Q^{0}$, the set $U^{\prime}=Q-\operatorname{cl}\left(Q^{0}-U\right)$ is open, nonnull, and homotopically trivial in $Q$. Hence, $h\left(U^{\prime}\right) \cdot Q^{0}=h(U-T)$ is nonnull, (relatively) open in $Q^{0}$, and homotopically trivial [3, Lemma 8.3], and thus $U-T$ is, also.

In the case of a separable, locally compact group, one may drop the hypothesis of Theorem 2 that the subset on which it acts be locally compact, as Theorem 3 below shows.

THEOREM 3. Any transformation group action of a separable, locally compact group on a closed set with property $Z$ in a separable, infinitedimensional Frechet space can be extended to an action of $G$ on the entire space.

Proof. Let $(G, X, v)$ be the transformation group under consideration. Without loss of generality, one may assume that the action is effective, that is, that if $g$ is in $G$ and is not the identity, then there is some $x$ in $X$ such that $v(g, x) \neq x$. Effectiveness implies, in this case, that $G$ is metrizable, for it must be Hausdorff and satisfy the first axiom of countability at each point.

As $G$ is separable, locally compact, and metrizable, the space $E$ of all continuous functions from $G$ into $l_{2}$ under the topology of uniform convergence on compacta is itself a separable, infinite-dimensional Frechet space. Furthermore, the homomorphism of $G$ into the group of linear homeomorphisms of $E$ onto itself defined in the proof of Theorem 1 in this case defines an action of $G$ on $E$ as a transformation group.

Let $f_{1}$ be an imbedding of $X$ in $l_{2}$ as a closed set, and let $f_{2}$ be the imbedding of $X$ in $E$ induced from $f_{1}$ as in the proof of Theorem 1 . Now, as before, the action of $G$ on $E$ extends the action of $G$ on $f_{2}(X)$ induced from $v$.

Let $Y^{\prime}=E \times Y$, where $Y$ is the separable, infinite-dimensional 
Frechet space containing $X$ which is in question. Now let the action of $G$ on $E$ generate an action of $G$ on $Y^{\prime}$, trivial in the $Y$ coordinate, as was done in the proof of Theorem 2. If $f_{3}: X \rightarrow Y^{\prime}$ is defined by $f_{3}(x)=\left(f_{2}(x), 0\right)$, then $f_{3}$ is an imbedding and $f_{3}(X)$ has property $Z$ in $Y^{\prime}$, as it lies in a subspace of infinite co-dimension. As the defined action of $G$ on $Y^{\prime}$ extends the induced action on $f_{3}(X)$ (induced from $v$ by $f_{3}$ ) and as $Y^{\prime}$ is a separable, infinite-dimensional Frechet space and is thus homeomorphic to $Y$, there remains only to apply Theorem $\mathrm{A}$ in order to complete the proof.

REMARK. Raymond Y.-T. Wong has proven in [12] (but not specifically stated) that if $X$ is a subset of the Hilbert cube $Q$ which is homeomorphic to $Q$ and is bi-collared (that is, there exists an open set $U$ in $Q$ containing $X$ which is homeomorphic to $X \times(-1,1)$ by a homeomorphism carrying each $x$ in $X$ to $(x, 0)$ ), then any action of a transformation group on $X$ can be extended to an action on $Q$. (Wong proved that there exists a homeomorphism of $Q$ onto itself carrying $X$ on to the set of all points of $Q$ whose first coordinate is 0 .) Wong has also found a Cantor set in $Q$ and a homeomorphism of it on to itself which cannot be extended to a homeomorphism of $Q$ onto itself [11]. These two results are the only ones of which the author is aware concerning the extension of transformation group actions on subsets of $Q$ which do not have property $Z$.

\section{REFERENCES}

1. R. D. Anderson, Hilbert space is homeomorphic to the countable infinite product of lines, Bull. Amer. Math. Soc. 72 (1966), 515-519.

2. - Topological properties of the Hilbert cube and the infinite product of open intervals, Trans. Amer. Math. Soc. 126 (1967), 200-216.

3. - On topological infinite deficiency, Michigan Math. J. 14 (1967), 365-383.

4. P. C. Baayen and J. de Groot, Linearization of locally compact transformation groups in Hilbert space, Mathematisch Centrum, Amsterdam, 1967.

5. C. Bessaga and A. Pełczynski, Some remarks on homeomorphisms of F-spaces, Bull. Acad. Polon. Sci. Sér. Sci. Math. Astronom. Phys. 10 (1962), 265-270.

6. M. I. Kadec, On topological equivalence of all separable Banach spaces, Dokl. Akad. Nauk. SSSR 167 (1966), 23-25 = Soviet Math. Dokl. 7 (1966), 319-322.

7. O.-H. Keller, Die Homeomorphie der kompakten konvexen Mengen im Hilbertschen Raum, Math. Ann. 105 (1931), 748-758.

8. J. L. Kelley, General topology, Van Nostrand, New York, 1955.

9. V. L. Klee, Jr., Some topological properties of convex sets, Trans. Amer. Math. Soc. 78 (1955), 30-45.

10. A. P. Robertson and W. J. Robertson, Topological vecțor spaces, Cambridge Univ. Press, New York, 1964.

11. R. Y.-T. Wong, A wild Cantor set in the Hilbert cube, Pacific J. Math. 24 (1968), 189-193.

12. —- Extending homeomorphisms by means of collaring (to appear).

The Institute For Advanced Study 